\title{
Crescimento de jundiá e carpa húngara criados em sistema de recirculação de água
}

\author{
Jundiá growth and Common Carp reared in recirculated water system
}

\author{
Viviani CorrêiaI João Radünz Neto ${ }^{\text {II }}$ Rafael Lazzari ${ }^{\text {III }}$ Cátia Aline Veiverberg ${ }^{\mathrm{I}}$ \\ Giovani Taffarel Bergamin ${ }^{\mathrm{I}}$ Fabio de Araújo Pedron ${ }^{\mathrm{I}}$ Cristiano Costenaro Ferreira ${ }^{\mathrm{IV}}$ \\ Tatiana Emanuelliv Cristiane Portes Ribeiro ${ }^{\mathrm{VI}}$
}

RESUMO

O presente estudo foi realizado com o objetivo de comparar o crescimento e o rendimento de carcaça e a composição corporal de juvenis de jundiá (Rhamdia quelen) $e$ carpa húngara (Cyprinus carpio) criados em sistema de recirculação de água, em mono e bicultivo, durante 60 dias, na fase de recria. Cinco tratamentos com três repetições foram testados: $100 \mathrm{C}=100 \%$ carpa; $100 \mathrm{~J}=100 \%$ jundiá; $50 \mathrm{C} 50 \mathrm{~J}=50 \%$ carpa $50 \%$ jundiá; $75 \mathrm{C} 25 \mathrm{~J}=75 \%$ carpa $25 \%$ jundiá e 25C75J=25\% carpa 75\% jundiá, sendo utilizados 20 peixes por unidade experimental. Foram avaliados o peso $(P)$, a biomassa total (BT), o fator de condição (FC), a conversão alimentar aparente (CAA), a taxa de crescimento específico (TCE), o rendimento de carcaça $(R C)$, o índice digestivosomático, o índice hepato-somático, o quociente intestinal, as composições centesimais dos peixes, as taxas de deposição de proteína e a gordura corporal. Não foram observadas diferenças no peso, no FC e na TCE dos jundiás $(P>0,05)$. Para essas variáveis, as carpas submetidas ao tratamento $25 \mathrm{C75J}$ apresentaram valores significativamente maiores $(P<0,05) . O$ tratamento $100 \mathrm{~J}$ produziu os melhores valores de CAA e BT e não diferiu do 25C75J. Em ambas as espécies, não houve diferença significativa para $R C$ éndices digestivos. Em bicultivo, a proporção $25 \%$ carpa $75 \%$ jundiá resulta no maior peso individual das carpas húngaras e na maior biomassa total.

Palavras-chave: biomassa, Cyprinus carpio, bicultivo, Rhamdia quelen, rendimento de carcaça.

\begin{abstract}
A study was conducted to evaluate growth, carcass yield and body composition of jundiá and common carp raised in mono or duoculture in a recirculated water system, for 60 days, during the growing. Five treatments were tested with three replicates: $100 \mathrm{C}=100 \%$ carp; $100 \mathrm{~J}=100 \%$ jundiá, $50 \mathrm{C} 50 \mathrm{~J}=50 \%$ carp $50 \%$ jundiá; $75 \mathrm{C} 25 \mathrm{~J}=75 \%$ carp $25 \%$ jundiá and 25C75J=25\% carp 75\% jundiá. Twenty fishes were distributed in each experimental unit. It was evaluated weight $(W)$, total biomass (TB), condition factor (CF), feed conversion rate (FCR), specific growth rate (SGR), carcass yield (YC), digestive-somatic index, hepatossomatic index, intestinal quotient and the proximate composition and body deposition of protein and fat. Considering jundiá performance, no statistical difference was observed for $W, C F$ and SGR $(P>0.05)$. Common carp from treatment $25 \mathrm{C} 75 \mathrm{~J}$ showed the highest values $(P<0.05)$ for these variables. The best FCR and TB values were obtained for $100 \mathrm{~J}$ and $25 \mathrm{C} 75 \mathrm{~J}$ treatments. In both species, there were no differences for carcass yield or digestive indexes. Thus, the treatment $25 \mathrm{C} 75 \mathrm{~J}$, composed by $25 \%$ carp and $75 \%$ jundiá presented the highest final weight for carp and total biomass.
\end{abstract}

Key words: biomass, Cyprinus carpio, polyculture, Rhamdia quelen, carcass yield.

\section{INTRODUÇÃO}

O sistema de bicultivo destaca-se por possibilitar a criação de mais de uma espécie de peixe

IPrograma de Pós-graduação em Zootecnia (PPGZ), Centro de Ciências Rurais (CCR), Universidade Federal de Santa Maria (UFSM), Santa Maria, RS, Brasil.

IILaboratório de Piscicultura, Departamento de Zootecnia, CCR, UFSM, 97010-120, Santa Maria, RS, Brasil. Email:jradunzneto@smail.ufsm.br. Autor para correspondência.

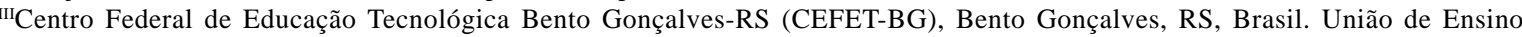
Superior de Diamantina (UNED), Santo Augusto, RS, Brasil.

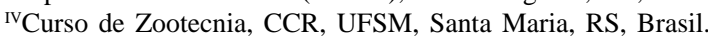

vopartamento de Tecnologia e Ciência dos Alimentos, CCR, UFSM, Santa Maria, RS, Brasil.

${ }^{\mathrm{VI} C}$ Curso de Farmácia e Bioquímica, UFSM, Santa Maria, RS, Brasil. 
no mesmo espaço de cultivo (PAPOUTSOGLOU et al., 1992). Nesse sistema, o crescimento e a produção das espécies podem ser maiores em comparação ao monocultivo devido às interações positivas entre elas (KESTEMONT, 1995), ou seja, algumas espécies têm seu desempenho melhorado na presença de outras (LUTZ, 2003). Isso foi observado no mono e bicultivo de carpa comum (Cyprinus carpio) e tilápia (Oreochromis niloticus), em sistema de recirculação de água, em que o melhor desempenho para ambas as espécies foi no bicultivo (40\% carpa, $60 \%$ tilápia), e a carpa obteve o pior crescimento em monocultivo (PAPOUTSOGLOU et al., 2001). Para se obter uma boa produção com esse tipo de criação, é essencial ter cuidado na combinação e proporção de cada espécie (JENA et al. 2002).

O jundiá (Rhamdia quelen) é uma espécie importante na piscicultura da região Sul do Brasil, pois, além de ser um peixe de fácil manejo, rápido crescimento e que se adapta bem às dietas elaboradas e variações do ambiente (CARNEIRO et al., 2002; FRACALOSSI et al., 2002), é considerado promissor para a criação intensiva, tendo boa aceitação pelos consumidores e maior valor comercial (FRACALOSSI et al., 2007; SILVA et al., 2008).

As carpas são o grupo de peixes mais cultivados no mundo, representando aproximadamente 40\% da produção (FAO, 2005). Dentre elas, está a carpa húngara (Cyprinus carpio), espécie de acelerado crescimento e fácil adaptação às condições de cativeiro (POLI et al., 2004). No entanto, essa espécie pode apresentar rejeição ao consumo pela população, devido a um sabor indesejável em sua carne, adquirido pelo hábito de revolver o fundo do tanque à procura de alimento.

O cultivo em sistema de recirculação de água elimina fatores como plâncton, algas e oscilações bruscas de temperatura, que podem influenciar a qualidade dos produtos obtidos. Assim, este trabalho visa obter informações de forma clara sobre a interação dessas espécies criadas em bicultivo, já que os dados são limitados nesse tipo de sistema (KARAKATSOULI et al., 2006; PAPOUTSOGLOU et al., 2001).

Com isso, o objetivo deste trabalho foi avaliar o desenvolvimento do jundiá e da carpa húngara, na fase de recria, mantidos em sistema de recirculação de água, usando mono e bicultivo.

\section{MATERIAL E MÉTODOS}

O experimento foi conduzido no Laboratório de Piscicultura da Universidade Federal de Santa Maria (altitude 95m, 294's, 534' $\mathrm{W}$ ), no período de setembro a novembro de 2007, totalizando 60 dias. Para a realização do experimento, utilizou-se um sistema com recirculação de água termorregulado, composto por 15 tanques experimentais de 2801 cada, com entrada e saída de água individual, com um reservatório principal (2000L) e dois filtros biológicos com pedra britada.

A análise de água foi realizada diariamente nos primeiros 15 dias de experimento. Após esse período, as análises foram feitas semanalmente. Os parâmetros medidos foram: oxigênio dissolvido (ppm), amônia total (ppm), nitrito (ppm), alcalinidade (mg $\mathrm{CaCo}_{3}$ $\left.\mathrm{L}^{-1}\right)$, pH e temperatura. Utilizou-se, para a medição do oxigênio, um oxímetro digital (YSI-Yellowsprings-USA) e, para a temperatura, um termômetro com bulbo de mercúrio. As demais análises foram realizadas por kit colorimétrico (Alfakit ${ }^{\circledR}$ ), o qual possibilita que a análise de nitrito seja realizada pelo método da alfa-naftilamina (segundo FRIES, 1971); para o pH, foi utilizado indicador conforme AWWA (1970); para a análise de amônia, foi utilizado o método colorimétrico Indotest; e a alcalinidade foi avaliada pelo método volumétrico de neutralização (ambos descritos no manual de análises da APHA(2005)).

Foram utilizados 300 peixes, na fase de recria, selecionados pelo comprimento total, tendo em vista que esse é o parâmetro utilizado no momento da comercialização de alevinos em pisciculturas. Os jundiás possuíam peso médio inicial de $12,45 \pm 0,11 \mathrm{~g}$ e comprimento de $10,68 \pm 0,01 \mathrm{~cm}$, já as carpas, 24,10 $\pm 0,66 \mathrm{~g}$ e $11,19 \pm 0,23 \mathrm{~cm}$ ), sendo distribuídos 20 peixes por unidade experimental. Os peixes passaram por período de adaptação de 30 dias antes do início do experimento.

Foram avaliados cinco tratamentos, sendo dois monocultivos: $100 \mathrm{C}=100 \%$ carpa; $100 \mathrm{~J}=100 \%$ jundiá e três bicultivos: $50 \mathrm{C} 50 \mathrm{~J}=50 \%$ carpa $50 \%$ jundiá; $75 \mathrm{C} 25 \mathrm{~J}=75 \%$ carpa 25\% jundiá e 25C75J=25\% carpa $75 \%$ jundiá. Foi ofertada a mesma dieta para todos os tratamentos, usando-se, como base protéica, o farelo de soja (36,5\%) e a farinha de carne e ossos suína (40,5\%). A composição bromatológica da ração foi: $41,6 \%$ de proteína bruta, $15,3 \%$ de extrato etéreo, 2,5\% de fibra bruta, $15,8 \%$ de matéria mineral e $24,9 \%$ de extrativos não-nitrogenados.

$\mathrm{O}$ alimento peletizado foi ofertado aos animais duas vezes ao dia (5\% da biomassa/dia), e a quantidade de ração foi ajustada ao peso (biomassa total de cada unidade) a cada 15 dias. Diariamente, foi realizada a sifonagem dos tanques às 8 e 16h, para a retirada dos resíduos e, em seguida, a ração foi ofertada.

Ao final do período experimental, os peixes foram submetidos a jejum de 24 horas e anestesiados em trifenoxietanol (0,03\%), para a realização da medição de peso e comprimento. Para avaliação do rendimento 
de carcaça e índices digestivos, três peixes por tratamento foram abatidos por hipotermia. Também foi abatido e triturado um peixe de cada espécie por repetição, para análises de composição centesimal do peixe inteiro. Essas amostras foram encaminhadas para o Núcleo Integrado de Análises Laboratoriais (NIDAL, UFSM). A proteína bruta, a umidade e as cinzas foram determinadas seguindo metodologias descritas na AOAC (1995), já a gordura foi extraída e quantificada com base no método de BLIGH \& DYER (1959).

A partir dos dados, foram obtidos os seguintes parâmetros: peso final (g); biomassa total (g); fator de condição: $\mathrm{FC}=$ peso $\times 100 /$ (comprimento total $)^{3}$; taxa de crescimento específico: TCE $(\% /$ dia $)=$ [(ln (peso final) - $\ln$ (peso inicial)) /dias] x 100; conversão alimentar aparente: $\mathrm{CAA}=$ alimento consumido/ganho em peso; rendimento de carcaça: RC $(\%)=$ peso do peixe sem as vísceras/peso do peixe inteiro; índice digestivo-somático: IDS $(\%)=$ (peso trato/peso corporal X 100); índice hepato-somático: IHS (\%) = (peso fígado/peso corporal X 100); quociente intestinal: $\mathrm{QI}=$ (comprimento trato/comprimento total); \% de gordura mesentérica (GM); deposição de proteína corporal (g): PBTD $=[\mathrm{Pf} *(\% \mathrm{PBCf} / 100)]-[\mathrm{Pi} *(\% \mathrm{PBCi} /$ $100)]$; e deposição de gordura corporal (g): GTD $=[\mathrm{Pf} *$ (\%GCf/100)]-[Pi* (\%GCi/100)].
Para realização das análises estatísticas, foi utilizado o software SAS ${ }^{\circledR}$ (2001). Os dados foram submetidos à teste de normalidade (Shapiro-Wilk) e análise de variância. As médias das variáveis zootécnicas foram ajustadas, por meio de análise de covariância, para a biomassa inicial de cada tanque, e comparadas pelo teste Pdiff $(\mathrm{P}<0,05)$. Os valores de carcaça, de composição centesimal e das taxas de deposição de proteína e gordura foram comparados pelo teste de Tukey. As variáveis que não apresentaram distribuição normal foram analisadas por ANOVA de Kruskall-Wallis.

\section{RESULTADOS E DISCUSSÃO}

Durante o período experimental, os parâmetros físico-químicos da água estiveram em níveis aceitáveis para peixes de águas temperadas (ARANA, 2004; BALDISSEROTTO \& SILVA, 2004). A temperatura média registrada foi de $22,11 \pm 0,81^{\circ} \mathrm{C}$, o oxigênio dissolvido foi de $5,73 \pm 0,20 \mathrm{ppm}$, a amônia total foi de $0,29 \pm 0,09 \mathrm{ppm}$, o nitrito foi de $0,11 \pm 0,06 \mathrm{ppm}$, o $\mathrm{pH}$ foi de 6,5 $\pm 0,0$ e a alcalinidade foi de $30,22 \pm 1,31 \mathrm{mg} \mathrm{CaCO}_{3} \mathrm{~L}^{-1}$.

O peso dos jundiás não diferiu significativamente entre os tratamentos (Figura 1). Esse resultado pode estar associado à desuniformidade entre

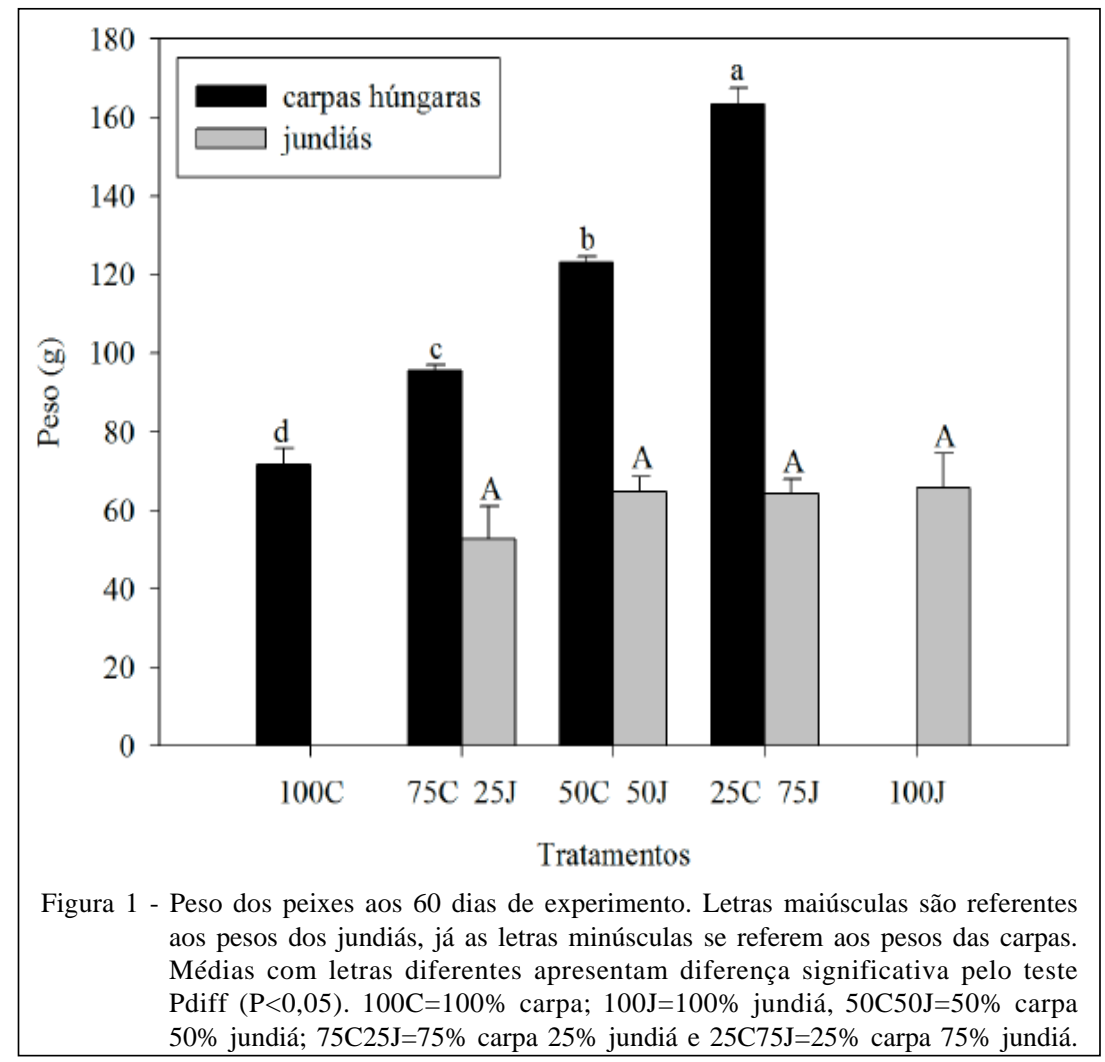

Ciência Rural, v.39, n.5, ago, 2009. 
os exemplares após 60 dias de cultivo. Já as carpas obtiveram maior peso individual no tratamento em que estavam em menor proporção (25C75J), apresentando o menor peso no monocultivo (Figura 1). Isso mostra o efeito positivo do bicultivo no crescimento dessa espécie quando associada ao jundiá. Resultados semelhantes foram encontrados no mono e bicultivo de carpa comum (Cyprinus carpio) e de tilápia (Oreochromis aureus), em sistema de recirculação de água, onde PAPOUTSOGLOU et al. (2001) verificaram que as duas espécies apresentaram maior peso individual na proporção com menor quantidade de carpas (40\%C 60\%T).
A maior biomassa total $(2027,72)$ foi obtida no tratamento 100J, que não diferiu da 25C75J (1956,35). Já a carpa em monocultivo obteve a menor biomassa $(1334,47)$, não diferindo do 75C25J $(1498,95)$. Entre os bicultivos, a maior biomassa obtida foi no tratamento 25C75J (Figura 2A). Desse modo, observa-se que o jundiá pode obter boa produção quando em monocultivo, enquanto a carpa atinge maior biomassa quando está em bicultivo com o jundiá e em menor quantidade na proporção. Isso confirma a proposta de JENA et al. (2002) de que a densidade de estocagem e a combinação/proporção de cada espécie terão influência na eficiência e produção nesse tipo de

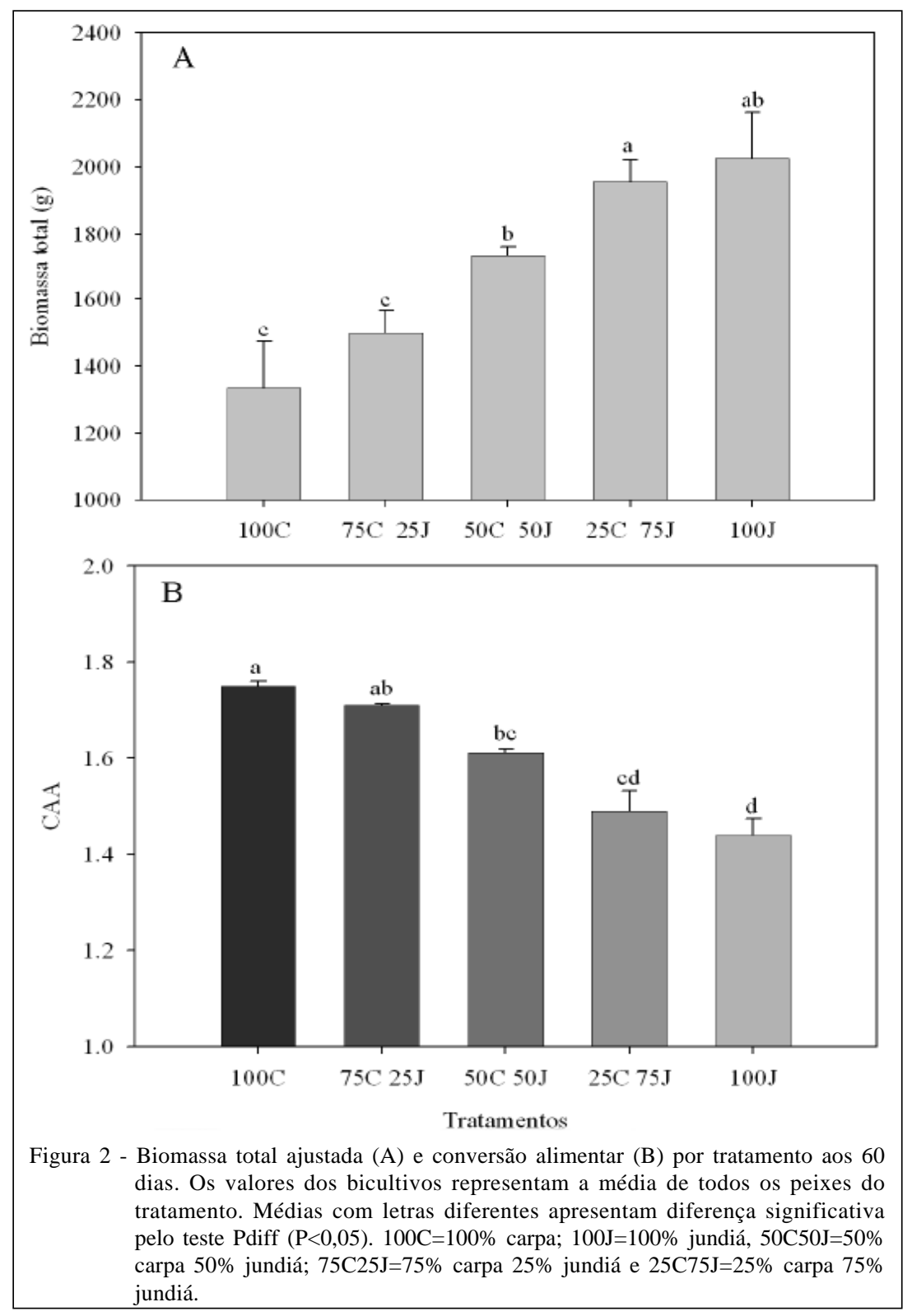

Ciência Rural, v.39, n.5, ago, 2009. 
criação. Também semelhante ao obtido no presente estudo, PAPOUTSOGLOU et al. (1992) observaram menor produção em monocultivo de carpa $\left(116 \mathrm{~kg} \mathrm{~m}^{-3}\right)$ e tilápia $\left(98,8 \mathrm{~kg} \mathrm{~m}^{-3}\right)$ do que nos bicultivos $60 \% \mathrm{C} 40 \% \mathrm{~T}$ $\left(126 \mathrm{~kg} \mathrm{~m}^{-3}\right)$ e $40 \% \mathrm{C} 60 \% \mathrm{~T}\left(132 \mathrm{~kg} \mathrm{~m}^{-3}\right)$.

O tratamento $100 \mathrm{~J}$ apresentou melhor conversão alimentar aparente $(\mathrm{CAA}=1,44)$, não diferindo do 25C75J $(1,49)$. Já os tratamentos 100C $(1,75)$ e 75C25J $(1,71)$ apresentaram as piores CAA (Figura 2B). Resultados semelhantes foram encontrados no bicultivo de carpa comum e tilápia, em que a melhor CAA $(1,44)$ foi no tratamento em que a carpa estava em menor percentagem (40\% carpa comum e 60\% tilápia), já na proporção $100 \%$ carpa comum foi observada a pior CAA $(1,73)$ (PAPOUTSOGLOU et al., 2001). Não foi observada diferença significativa $(\mathrm{P}>0,05)$ na taxa de crescimento específico (TCE) e no fator de condição (FC) para o jundiá (Tabela 1). No entanto, a carpa húngara obteve, tanto para TCE (1,92-3,25\%/dia), quanto para o FC $(1,83-2,65)$, os maiores valores no tratamento 25C75J e os menores valores em monocultivo (Tabela 1). Resultado similar foi obtido por PAPOUTSOGLOU et al. (2001), em que a carpa comum apresentou baixa TCE $(0,88)$ em monocultivo.

Com esses resultados até então apresentados (peso, biomassa, CAA, TCE e FC), percebe-se que o jundiá em monocultivo obtém boa produção e CAA; entretanto, a carpa húngara apresentou melhor desempenho no bicultivo 25C75J. Considerando que os dados foram obtidos em cultivo com água limpa e alimentação exclusiva com ração, pode-se inferir que, no caso de cultivo em tanques de terra, com a disponibilidade de alimento natural, esses resultados sejam ainda melhores.

Não houve diferença significativa $(\mathrm{P}>0,05)$ entre os tratamentos para rendimento de carcaça (8387\%), em ambas as espécies (Tabela 1). Valores entre 80-87\% de rendimento de carcaça também foram obtidos por LOSEKANN et al. (2008), confirmando o bom potencial do jundiá para a produção de carne. Para índice digestivo-somático (IDS), índice hepatosomático (IHS) e quociente intestinal (QI), não houve diferença significativa em ambas as espécies (Tabela 1). Dessa forma, provavelmente esses parâmetros não são influenciados pela forma de cultivo, mas estão mais relacionados à composição de dietas.

Com relação à composição do peixe inteiro, a umidade para o jundiá foi menor no tratamento 25C75J (Tabela 2), porém este apresentou maior gordura total depositada, enquanto que a menor gordura total foi observada no 75C 25J. Diante disso, é possível que tenha ocorrido disputas territoriais e/ou por alimento

Tabela 1 - Parâmetros produtivos, rendimento de carcaça e parâmetros digestivos por espécie, aos 60 dias experimentais

\begin{tabular}{|c|c|c|c|c|c|c|}
\hline & \multicolumn{4}{|c|}{--------------------------------------Tratamentos"------------------------------------- } & \multirow{2}{*}{$\mathrm{dpr}$} & \multirow{2}{*}{$\mathrm{P}$} \\
\hline & $100 \mathrm{C}$ ou $100 \mathrm{~J}$ & $50 \mathrm{C} 50 \mathrm{~J}$ & $75 \mathrm{C} 25 \mathrm{~J}$ & $25 \mathrm{C} 75 \mathrm{~J}$ & & \\
\hline \multicolumn{7}{|c|}{ Carpa húngara } \\
\hline $\mathrm{RC}(\%)^{1}$ & 86,15 & 85,19 & 83,21 & 84,84 & 2,17 & NS \\
\hline IDS (\%) & 3,45 & 3,64 & 3,27 & 3,82 & 0,66 & NS \\
\hline IHS (\%) & 2,35 & 2,51 & 1,96 & 2,95 & 0,47 & NS \\
\hline QI & 1,93 & 2,06 & 1,84 & 2,20 & 0,15 & NS \\
\hline GM $(\%)^{1}$ & 1,24 & 1,73 & 0,25 & 0,29 & 0,32 & $* *$ \\
\hline FC & $1,83^{\mathrm{c}}$ & $2,30^{\mathrm{bc}}$ & $2,10^{\mathrm{bc}}$ & $2,65^{\mathrm{a}}$ & 0,07 & $* *$ \\
\hline TCE (\%/dia) & $1,92^{\mathrm{d}}$ & $2,64^{\mathrm{b}}$ & $2,25^{c}$ & $3,25^{\mathrm{a}}$ & 0,02 & $* * *$ \\
\hline \multicolumn{7}{|c|}{ Jundiá } \\
\hline RC (\%) & 86,50 & 84,81 & 87,08 & 85,83 & 1,58 & NS \\
\hline IDS (\%) & 3,15 & 2,61 & 2,17 & 2,76 & 0,39 & NS \\
\hline IHS (\%) & 1,38 & 1,27 & 1,11 & 1,55 & 0,23 & NS \\
\hline QI & 1,25 & 1,15 & 1,15 & 1,19 & 0,18 & NS \\
\hline GM (\%) & 1,71 & 2,01 & 1,38 & 2,02 & 0,82 & NS \\
\hline $\mathrm{FC}^{1}$ & 1,06 & 1,01 & 0,91 & 1,03 & 0,07 & NS \\
\hline TCE (\%/dia) & 2,69 & 2,75 & 2,50 & 2,70 & 0,11 & NS \\
\hline
\end{tabular}

Médias que não possuem pelo menos uma letra em comum, na linha, apresentam diferença significativa pelo teste de Tukey ( $\mathrm{P}<0,05)$. dpr: desvio padrão residual; RC: rendimento de carcaça; IDS: índice digestivo-somático; IHS: índice hepato-somático; QI: quociente intestinal; GM: gordura mesentérica; FC: fator de condição; TCE: taxa de crescimento específico; $* * \mathrm{P}<0,01 ; * * * \mathrm{P}<0,0001$. NS=não significativo $(\mathrm{P}>0,05) .{ }^{1}$ ANOVA de Kruskall-Wallis "Tratamentos: $100 \mathrm{C}=100 \%$ carpa; $100 \mathrm{~J}=100 \%$ jundiá, 50C $50 \mathrm{~J}=50 \%$ carpa $50 \%$ jundiá; $75 \mathrm{C} 25 \mathrm{~J}=75 \%$ carpa $25 \%$ jundiá e $25 \mathrm{C} 75 \mathrm{~J}=25 \%$ carpa $75 \%$ jundiá. 
Tabela 2 - Composição centesimal (\%), taxas de deposição de proteína e gordura do peixe inteiro por espécie, aos 60 dias experimentais, expressas na matéria natural.

\begin{tabular}{|c|c|c|c|c|c|c|}
\hline & \multicolumn{4}{|c|}{ Tratamentos $^{\#}$} & \multirow[t]{2}{*}{$\mathrm{dpr}^{1}$} & \multirow[t]{2}{*}{$\mathrm{P}$} \\
\hline & $100 \mathrm{~J}$ ou $100 \mathrm{C}$ & $50 \mathrm{C} 50 \mathrm{~J}$ & $75 \mathrm{C} \mathrm{25J}$ & $25 \mathrm{C} 75 \mathrm{~J}$ & & \\
\hline \multicolumn{7}{|c|}{ Carpa húngara } \\
\hline Umidade & 72,63 & 71,19 & 72,91 & 71,90 & 0,72 & NS \\
\hline $\mathrm{Cinza}^{2}$ & 1,86 & 1,60 & 1,65 & 1,82 & 0,19 & NS \\
\hline Proteína & $17,25^{\mathrm{a}}$ & $15,50^{\mathrm{ab}}$ & $14,76^{\mathrm{b}}$ & $13,87^{b}$ & 0,72 & $* *$ \\
\hline Gordura & 10,27 & 12,99 & 11,30 & 11,12 & 1,15 & NS \\
\hline PBTD (g) & $13,85^{\mathrm{ab}}$ & $15,61^{\mathrm{ab}}$ & $12,38^{\mathrm{b}}$ & $16,39^{\mathrm{a}}$ & 1,33 & $*$ \\
\hline GTD (g) & $8,18^{\mathrm{b}}$ & $13,19^{\mathrm{a}}$ & $10,06^{\mathrm{ab}}$ & $13,26^{\mathrm{a}}$ & 1,27 & $* *$ \\
\hline \multicolumn{7}{|c|}{ Jundiá } \\
\hline Umidade & $71,40^{\mathrm{a}}$ & $72,99^{\mathrm{a}}$ & $72,48^{\mathrm{a}}$ & $67,48^{\mathrm{b}}$ & 1,37 & $* *$ \\
\hline Cinza & 3,06 & 2,96 & 2,95 & 2,69 & 0,29 & NS \\
\hline Proteína & 14,51 & 17,53 & 15,97 & 14,86 & 1,63 & NS \\
\hline Gordura $^{2}$ & 10,68 & 9,30 & 9,72 & 13,38 & 1,66 & NS \\
\hline PBTD (g) & 8,44 & 9,30 & 6,49 & 8,04 & 1,33 & NS \\
\hline GTD (g) & $6,23^{\mathrm{ab}}$ & $4,39^{\mathrm{ab}}$ & $3,68^{\mathrm{b}}$ & $7,40^{\mathrm{a}}$ & 1,15 & $*$ \\
\hline
\end{tabular}

Médias que não possuem pelo menos uma letra em comum, na linha, apresentam diferença significativa pelo teste de Tukey (P<0,05). ${ }^{1} \mathrm{dpr}$ : desvio padrão residual;

PBTD: proteína bruta total depositada; GTD: gordura total depositada;

$* \mathrm{P}<0,05$. $* * \mathrm{P}<0,01$. NS=não significativo $(\mathrm{P}>0,05) .{ }^{2}$ ANOVA de Kruskall-Wallis

"Tratamentos: $100 \mathrm{C}=100 \%$ carpa; $100 \mathrm{~J}=100 \%$ jundiá, 50C50J=50\% carpa 50\% jundiá; 75C25J=75\% carpa 25\% jundiá e 25C75J=25\% carpa 75\% jundiá.

entre as espécies quando a carpa estava em maior proporção, fazendo com que o jundiá tivesse maior gasto energético e, consequentemente, menor deposição de gordura corporal. No entanto, como os jundiás não apresentaram diferença para o parâmetro peso, acredita-se que não houve dominância das carpas na hora da alimentação. Além disso, uma menor deposição de gordura corporal é um excelente resultado tratando-se de rendimento de carcaça e qualidade de carne.

A carpa húngara obteve maior acúmulo de proteína no peixe inteiro em $100 \mathrm{C}$ que no $75 \mathrm{C} 25 \mathrm{~J}$ e 25C75J, e a proteína total depositada foi maior no tratamento 25C75J, diferindo do 75C25J (Tabela 2). No entanto, a gordura total depositada foi maior nos tratamentos 25C75J e 50C50J que no 100C (Tabela 2), assim como apresentou maior percentagem de gordura mesentérica no tratamento 50C50J (Tabela 1). Com isso, percebe-se que, embora a carpa tenha apresentado pior desempenho no tratamento 100C, obteve nessa proporção maior teor de proteína no peixe inteiro e menor deposição de gordura. Segundo GERI et al. (1995), muitos fatores podem influenciar a composição química dos peixes, como o meio ambiente, a idade do animal e a espécie. No momento da venda, o importante, para o produtor, é o peso do peixe; entretanto, para a indústria, o interessante é a relação entre as partes comestíveis que constituem o produto (CARNEIRO et al., 2004).

Devido à constante busca do aumento de produtividade com redução de custos, pesquisas devem ser desenvolvidas sobre a composição de dietas e a forma de cultivo. Além disso, diante da importância da criação de espécies consorciadas, são necessários mais estudos sobre as interações entre elas, que terão influência no crescimento, no metabolismo e na composição corporal desses animais.

\section{CONCLUSÃO}

O jundiá permite maior biomassa total que a carpa húngara em situação de monocultivo. Entre os bicultivos, a proporção de $25 \%$ carpa e $75 \%$ jundiá resulta em maior produção de biomassa total e maior peso individual da carpa húngara. Os parâmetros digestivos e os rendimentos de carcaça dos peixes não sofrem influência das diferentes proporções testadas no presente trabalho.

\section{REFERÊNCIAS}

AOAC. (Association of Official Analytical Chemists). Official Methods of Analysis of AOAC. 16.ed. Arlington, Virginia, 1995. 1018p.

Ciência Rural, v.39, n.5, ago, 2009. 
APHA (American Public Health Association). Standard Methods for the Examination of Water and Waste water). 21.ed. Washington, DC, 2005. 1368p.

ARANA, L.V. Princípios químicos de qualidade da água em aqüicultura: uma revisão para peixes e camarões. Florianópolis: UFSC, 2004. 231p.

AWWA (American Water Works Association). Processos simplificados para exame e análise da água. São Paulo, 1970. 276p.

BALDISSEROTTO, B.; SILVA, L.V.F. Qualidade da água. In: BALDISSEROTTO, B.; RADÜNZ NETO, J. Criação do jundiá. Santa Maria:UFSM, 2004. p.73-94.

BLIGH, E.G.; DYER, W.J. A rapid method of total lipid extraction and purification. Canadian Journal of Biochemistry and Physiology, v.37, n.8, p.911-917, 1959.

CARNEIRO, P.C.F. et al. Jundiá: um grande peixe para a região sul. Panorama da Aqüicultura, v.12, n.69, p.41-46, 2002.

CARNEIRO, P.C.F. et al. Processamento do jundiá Rhamdia quelen: rendimento de carcaça. Revista Acadêmica: Ciências Agrárias e Ambientais, v.2, n.3, p.11-17, 2004.

FAO - Food Agriculture Organization (FAO). Estatísticas de produção de peixes e pesca-2005. Disponível em: http:// www.fao.org/fi/statist/ On line. Capturado em 08 de janeiro de 2008.

FRACALOSSI, D.M. et al. No rastro das espécies nativas. Panorama da Aqüicultura, v.12, p.43-49, 2002.

FRACALOSSI, D.M. et al. O mito da onivoria do Jundiá Panorama da Aqüicultura, v.17, n.100, p.36-40, 2007.

FRIES, J. Análisis de trazas: métodos fotométricos comprobados. Darmstadt: Merck, 1971. 184p.

GERI, G. et al. Body traits and chemical composition of muscle in the common carp (Cyprinus carpio L.) as influenced by age and rearing environment. Aquaculture, v.129, p.329-333, 1995. Disponível em: <http:// www.sciencedirect.comscience?_ob=MImg\&_imagekey=B6T4D$3 X Y 2$ K2W-T-1\&_cdi $=4972 \&$ _user $=687358 \&$ \&orig $=$ bro wse \& coverDate $=01 \% 2 F 31 \quad \% 2 F 1995 \&$ sk $=9987099$ $98 \&$ view $=c \& w c h p=d G L z V l z-z S k z k \& m d 5=120 \mathrm{dc} 70275 \mathrm{~d} 8 \mathrm{a}$ 299f8c7745cc0de8888\&ie>. Doi: 10.1016/0044-8486(94)00300-D.

JENA, J.K. et al. Evaluation of production performance in carp polyculture with different stocking densities and species combinations. Journal Applied Ichthyology, v.18, p.165-171,
2002. Disponível em: < http://www3.interscience.wiley.com/cgi-bin/ fulltext/118914880>. Doi: 10.1046/j.1439-0426.2002.00302.x.

KARAKATSOULI, N. et al. Mono- and duoculture of juvenile sharpsnout seabream Diplodus puntazzo (Cetti) and gilthead seabream Sparus aurata L. in a recirculated water system. Aquaculture Research, v.37, p.1654-1661, 2006. Disponível em: <http://www3.interscience.wiley.com/cgi-bin/fulltext/ 118633520>. Doi: 10.1111/j.1365-2109.2006.01612.x.

KESTEMONT, P. Different systems of carp production and their impacts on the environment. Aquaculture, v.129, p.347372, 1995. Disponível em: <http://www.sciencedirect.com/ science?_ob=MImg\&_imagekey=B6T4D-3XY2K2W-J$1 \&$ \&_c di $=4972 \&$ \&_user $=687358$ \&_orig = bro wse \&_cover Date $=01 \% 2$ F $31 \% 2$ F $1995 \&$ $\mathrm{sk}=998709998 \& \mathrm{vi}$ i w $=\mathrm{c} \& \mathrm{w} \mathrm{chp}=\mathrm{d} \mathrm{GL} \mathrm{bVlW}$ zSkzV\&md5=3d9785d6ae01cb14e00cbdf6d1885387\&ie>. Doi: 10.1016/0044-8486(94)00292-V.

LOSEKANN, M.E. et al. Alimentação do jundiá com dietas contendo óleos de arroz, canola ou soja. Ciência Rural, v.38, n.1, p.225-230, 2008. Disponível em: <http:// www.scielo.br/scielo. php?script=sci_arttext\&pid=S0103$84782008000100036 \& \operatorname{lng}=$ pt $\& n r m=i s o>$. Doi: $10.1590 /$ S0103-84782008000100036.

LUTZ, C.G. Polyculture: principles, practices, problems and promise. Aquaculture Magazine, v.29, n.2, p.34-39, 2003.

PAPOUTSOGLOU S.E. et al. Polyculture rearing of Cyprinus carpio (L.) and Oreochromis aureus (St.) using a closed circulated system. Aquaculture, v.103, p.311-320, 1992.

PAPOUTSOGLOU S.E. et al. Growth and physiological changes in scaled carp and blue tilapia under behavioural stress in mono and polyculture rearing using a recirculated water system. Aquaculture International, v.9, p.509-518, 2001. Disponível em: <http:// www.springerlink.com.w10050.dotlib.com.br/content/ n71p386520n714n4?p=4df164411d814f00bf202c52f4e67b34\&pi=4> . Doi: 10.1023/A:1020506814609.

POLI, C.R. et al. Aqüicultura: experiências brasileiras. Florianópolis: Multitarefa, 2004. 456p.

SAS. Statistical Analysis System. User's Guide. Version 8.02. North Caroline, 2001. 3864p.

SILVA, L.B. et al. Introduction of jundia Rhamdia quelen (Quoy \& Gaimard) and Nile tilapia Oreochromis niloticus (Linnaeus) increases the productivity of carp polyculture in southern Brazil. Aquaculture Research, v.1, p.1-10, 2008. Disponível em: <http://www3.interscience.wiley.com/cgi-bin/fulltext/ 119417015>. Doi: 10.1111/j.1365-2109.2008.01912.x. 